\title{
Convergence to Diffusion Waves of Solutions for Viscous Conservation Laws
}

\author{
I.-Liang Chern ${ }^{1, \star}$ and Tai-Ping Liu $^{2, \star \star}$ \\ 1 Institute of Mathematics, Academia Sinica, Taiwan, R.O.C. \\ ${ }^{2}$ Department of Mathematics, University of Maryland, College Park, MD 20742, USA
}

\begin{abstract}
We study the large-time behavior of solutions of viscous conservation laws. It is shown that solutions tend to diffusion waves, which are constructed based on the heat equation and Burgers equation. The convergence is in the $L_{p}, 1 \leqq p \leqq \infty$ sense and is obtained as a consequence of the $L_{2}$ decay of the difference between the solution and its asymptotic state of diffusion waves.
\end{abstract}

\section{Introduction}

Consider a system of viscous conservation laws

$$
\frac{\partial u}{\partial t}+\frac{\partial f(u)}{\partial x}=\frac{\partial}{\partial x}\left(B(u) \frac{\partial u}{\partial x}\right), \quad u \in \mathbb{R}^{n}, \quad t \geqq 0, \quad-\infty x<\infty .
$$

We are interested in the large-time behavior of solutions whose initial value tends to a constant state at $x= \pm \infty$. Without loss of generality we take the constant state to be zero:

$$
u(x, 0) \rightarrow 0 \quad \text { as } \quad x \rightarrow \pm \infty .
$$

Physical models of the form (1.1) include the compressible Navier-Stokes equations and magnetohydrodynamics. The $n \times n$ viscosity matrix $B(u)$ represents a dissipative mechanism, and the solution $u(x, t)$ is expected to decay to the zero state as $t \rightarrow \infty$. The decay in $L_{\infty}$ and $L_{2}$ has been studied by viewing (1.1) as a perturbation of the linearized equations

$$
\frac{\partial u}{\partial t}+f^{\prime}(0) \frac{\partial u}{\partial x}=B(0) \frac{\partial^{2} u}{\partial x^{2}}
$$

\footnotetext{
* Supported by the National Sciences Council of the Republic of China under the contract NSC76-0208-M-001-09

* Supported by NSF under Grant No. DMS-84-01355
} 
$[2,7]$, and references therein. The purpose of the present article is to study the large-time behavior in the $L_{p}$ sense $1 \leqq p \leqq \infty$. System (1.1) is in conservation form

$$
\int_{-\infty}^{\infty} u(x, t) d x=\int_{-\infty}^{\infty} u(x, 0) d x, \quad t \geqq 0 .
$$

Consequently $u(x, t)$ does not decay in $L_{1}$. The study of $L_{1}$ behavior of $u(x, t)$ involves nonlinear waves. The asymptotic state has been constructed in [5] based on the self-similar solutions of the heat equation and Burgers equation:

$$
\begin{gathered}
\frac{\partial w}{\partial t}+c \frac{\partial w}{\partial x}=\alpha \frac{\partial^{2} w}{\partial x^{2}}, \\
\frac{\partial w}{\partial t}+c \frac{\partial w}{\partial x}+d w \frac{\partial w}{\partial x}=\alpha \frac{\partial^{2} w}{\partial x^{2}},
\end{gathered}
$$

where $c, d, \alpha>0$ are constant and $w \in \mathbb{R}^{1}$. Our main result shows that $u(x, t)$ tends to its asymptotic state in $L_{1}$ as $t \rightarrow \infty$. The result yields the distribution of the basic physical density $u(x, t)$, and therefore is of physical significance. The phenomenon of decay in $L_{2}$ and $L_{\infty}$ for solutions of the nonlinear system (1.1) is similar to that for solutions of the linearized system (1.3). On the other hand, $L_{1}$ behavior for (1.1) involves nonlinear waves, while for (1.3) involves only linear waves.

To simplify the presentation we carry out our analysis for the simplified situation where the viscosity $B(u)$ is the identity matrix:

$$
\frac{\partial u}{\partial t}+\frac{\partial f(u)}{\partial x}=\frac{1}{2} \frac{\partial^{2} u}{\partial x^{2}} .
$$

Our main assumption is that the associated inviscid conservation laws

$$
\frac{\partial u}{\partial t}+\frac{\partial f(u)}{\partial x}=0
$$

are strictly hyperbolic. This means that $f^{\prime}(u)$ has real and distinct eigenvalues $\lambda_{1}(u)$ $<\lambda_{2}(u)<\ldots<\lambda_{n}(u)$ with right eigenvectors $r_{i}(u)$ and left eigenvectors $l_{i}(u)$, $i=1,2, \ldots, n$. By a linear transformation we may assume that

$$
f^{\prime}(0)=\left\langle\begin{array}{cc}
\lambda_{1}(0) & 0 \\
0 & \lambda_{n}(0)
\end{array}\right\rangle, \quad \begin{aligned}
& r_{i}(0)=\left(\delta_{i 1}, \ldots, \delta_{i n}\right)^{t} \\
& l_{i}(0)=\left(\delta_{i 1}, \ldots, \delta_{i n}\right) .
\end{aligned}
$$

We abbreviate $\lambda_{i}(0), r_{i}(0)$ by $\lambda_{i}, r_{i}$ and decompose the initial data into

$$
u(x, 0)=\sum_{i=1}^{n} \theta_{i}^{0}(x) r_{i}=\left(\theta_{1}^{0}(x), \theta_{2}^{0}(x), \ldots, \theta_{n}^{0}(x)\right) .
$$

For each mode $i$, we consider the parabolic equation

$$
\frac{\partial \theta_{i}}{\partial t}+\lambda_{i} \frac{\partial \theta_{i}}{\partial x}+\frac{1}{2} b_{i} \frac{\partial\left(\theta_{i}^{2}\right)}{\partial x}=\frac{1}{2} \frac{\partial^{2} \theta_{i}}{\partial x^{2}}, \quad b_{i} \equiv \nabla \lambda_{i}(0) \cdot r_{i}(0),
$$

with initial data

$$
\theta_{i}(x, 0)=\theta_{i}^{0}(x)
$$


When $b_{i}=0,(1.11)$ is the linear heat equation, and the solution is

$$
\theta_{i}(x, t)=\frac{1}{\sqrt{2 \pi t}} \int_{-\infty} e^{-\frac{\left(x-\lambda_{i} t-y\right)^{2}}{2 t}} \theta_{i}^{0}(y) d y, \quad b_{i}=0 .
$$

When $b_{i} \neq 0,(1.11)$ is the Burgers equation which can be linearized through the Hopf-Cole transformation, [1],

$$
\begin{gathered}
\frac{\partial \varphi_{i}}{\partial t}+\lambda_{i} \frac{\partial \varphi_{i}}{\partial x}=\frac{1}{2} \frac{\partial^{2} \varphi_{i}}{\partial x^{2}}, \\
\varphi_{i}(x, t) \equiv \exp \left(-b_{i} \int \theta_{i}(\xi, t) d \xi\right),
\end{gathered}
$$

and the solution of (1.11) and (1.12) is

$$
\begin{aligned}
\theta_{i}(x, t) & =-\frac{1}{b_{i}}\left(\ln \varphi_{i}\right)_{x}, \\
\varphi_{i}(x, t) & =\frac{1}{\sqrt{2 \pi t}} \int_{-\infty}^{\infty} e^{\frac{-\left(x-\lambda_{z} t-y\right)^{2}}{2 t}} \varphi_{i}^{0}(y) d y, \\
\varphi_{i}^{0}(y) & \equiv \exp \left(-b_{i} \int^{x} \theta_{i}^{0}(\eta) d y\right), \quad b_{i} \neq 0 .
\end{aligned}
$$

The solutions (1.13) and (1.14) are called linear and nonlinear diffusion waves, respectively, and will be shown to represent the asymptotic state of the solution of (1.7) and (1.10). The construction of diffusion waves here differs somewhat from that of [5].

Our basic assumption on the initial data $u(x, 0)$ is that

$$
\int_{-\infty}^{\infty}\left|\theta_{i}^{0}(x)\right| d x<\infty, \quad \int_{-\infty}^{\infty}\left|x \theta_{i}^{0}(x)\right| d x<\infty .
$$

The main result of this paper is to show that the solution $u(x, t)$ of $(1.7)$ and (1.10) converges to $\theta(x, t) \equiv\left(\theta_{1}(x, t), \ldots, \theta_{n}(x, t)\right)$ as $t \rightarrow \infty$. Note that both (1.7) and (1.11) are conservation laws. Thus from (1.10) we have

$$
\begin{gathered}
\int_{-\infty}^{\infty} \bar{u}(x, t) d x=0, \quad t \geqq 0, \\
\bar{u}(x, t) \equiv u(x, t)-\theta(x, t),
\end{gathered}
$$

and it is natural to introduce

$$
v(x, t) \equiv \int_{-\infty}^{x} \bar{u}(y, t) d y
$$

We have from (1.17), (1.10), and (1.11) that

$$
\begin{aligned}
& \frac{\partial \bar{u}}{\partial t}+f^{\prime}(0) \frac{\partial \bar{u}}{\partial x}+\frac{\partial}{\partial x} L(\theta) \bar{u}+\frac{\partial N(\theta, \bar{u})}{\partial x}=\frac{1}{2} \frac{\partial^{2} \bar{u}}{\partial x^{2}}, \\
& \frac{\partial v}{\partial t}+f^{\prime}(0) \frac{\partial v}{\partial x}+L(\theta) \frac{\partial v}{\partial x}+N\left(\theta, \frac{\partial v}{\partial x}\right)=\frac{1}{2} \frac{\partial^{2} v}{\partial x^{2}},
\end{aligned}
$$




$$
\begin{gathered}
\bar{u}(x, 0)=v(x, 0)=0, \\
L(\theta) \equiv f^{\prime \prime}(0)(\theta), \\
N(a, b) \equiv f(a+b)-f^{\prime}(0)(a+b)-\frac{1}{2} \sum_{i=1}^{n}\left(f^{\prime}(0) r_{i}, r_{i}\right) a_{i}^{2}-\left(f^{\prime}(0) a, b\right) .
\end{gathered}
$$

We carry out our analysis based on (1.20). The first result concerns the $L_{2}$ decay of $v(x, t)$ :

Theorem 1.1. Suppose that the initial data $u(x, 0)$ has the property that

$$
\delta \equiv \int_{-\infty}^{\infty}(|u(x, 0)|+|x u(x, 0)|) d x+\int_{-\infty}^{\infty}\left(|u(x, 0)|^{2}+\left|u_{x}(x, 0)\right|^{2}\right) d x
$$

is sufficiently small. Then the solution $v(x, t)$ of $(1.20),(1.21)$ exists in $c\left((0, \infty), H^{l+1}\right)$ $\cap L^{2}\left((0, \infty), H^{l+2}\right)$ and satisfies for all $\sigma>0$ sufficiently small,

$$
\left\|D^{l} v(t)\right\|_{L_{2}}=O(1) \delta(1+t)^{-1 / 4-l / 2+\sigma}, \quad l=0,1,2, \ldots .
$$

Here $H^{l}$ is the Sobolev space, $H^{0}=L_{2}$ and $D^{l} \equiv \partial^{l} / \partial x^{l}$.

As a consequence of the above decay result for $v(x, t)$, we obtain the asymptotic behavior of $u(x, t)$. We show that $u(x, t)$ tends to $\theta(x, t)$. It can be shown that $\theta(x, t)$ in turn converges to the combination of linear and nonlinear heat kernels:

$$
\begin{gathered}
\theta^{*}(x, t) \equiv \sum_{i=1}^{n} t^{-1 / 2} \theta_{i}^{*}\left(\frac{x-\lambda_{i} t}{\sqrt{t}}\right) r_{i}, \\
\theta_{i}^{*}(\bar{x})\left\{\begin{array}{l}
m_{i} \\
\sqrt{2 \pi} e^{-\frac{\bar{x}^{2}}{2}} \quad \text { if } \quad b_{i}=0 \\
1 \\
=-\frac{b_{i}\left(\ln \psi_{i}(\bar{x})\right)^{\prime}}{\text { if }} b_{i} \neq 0
\end{array}\right. \\
\psi_{i}(\bar{x}) \equiv e^{\frac{b_{i} m_{i}}{2}} \int_{-\infty}^{\bar{x}} e^{-y^{2} / 2} d y+e^{\frac{-b_{i} m_{z}}{2}} \int_{\bar{x}}^{\infty} e^{-\frac{y^{2}}{2} d y}, \\
m_{i} \equiv \int_{-\infty}^{\infty} \theta_{i}(x, 0) d x .
\end{gathered}
$$

Theorem 1.2. Under the same hypotheses as in Theorem 1.1, the solution of (1.7),(1.2) exists in $C\left((0, \infty), H^{l}\right) \cap L^{2}\left((0, \infty), H^{l+1}\right)$ and satisfies, for any $\sigma>0$ sufficiently small

$$
\left\|D^{l}\left(u-\theta^{*}\right)\right\|_{L_{p}}(t)=O(1) \delta t^{-\left(\frac{l}{2}+1-\frac{1}{2 p}+\sigma\right)} \text {. }
$$

Of particular interest is the case where $p=1$ and $p=\infty$. From the conservation laws (1.1) we have

$$
\int_{-\infty}^{\infty} u(x, t) d x=\int_{-\infty}^{\infty} u(x, 0) d x, \quad t \geqq 0
$$


and so the solution $u(x, t)$ in general does not decay in $L_{1}$. Because of (1.16), we have the decay of $u-\theta^{*}$ in $L_{1}$ :

$$
\int_{-\infty}^{\infty}\left|u(x, t)-\theta^{*}(x, t)\right| d x=O(1) \delta t^{-\frac{1}{2}+\sigma} .
$$

About the convergence in $L_{\infty}$, we have the following more precise formulation in the self-similar coordinates:

$$
\begin{gathered}
\sup _{\bar{x} \in I_{i}(t)}\left[t^{1 / 2} u\left(\lambda_{i} t+\sqrt{t} \bar{x}, t\right)-\theta_{i}^{*}(\bar{x}) r_{i}\right]=O(1) t^{-\frac{1}{2}+\sigma}, \\
I_{i}(t) \equiv\left(\bar{x}:\left(\lambda_{i-1}-\lambda_{i}\right) t / 2 \leqq \sqrt{t} \bar{x} \leqq\left(\lambda_{i+1}-\lambda_{i}\right) t / 2\right), \\
\lambda_{0} \equiv-\infty, \quad \lambda_{n+1} \equiv \infty .
\end{gathered}
$$

In Sect. 2, we make basic estimates on diffusion waves. In Sect. 3 we first use Moser's inequalities to estimate the nonlinear terms in (1.20). This allows us to prove the decay result, Theorem 1.1, by employing an analysis of Kawashima [2] on the energy decay for weakly nonlinear systems. Theorem 1.2 is proved in Sect. 4 as a corollary of Theorem 1.1. Finally in Sect. 5 we indicate how to generalize our analysis for (1.7) to the general system (1.1). This will involve linear hyperbolic waves in the construction of diffusion waves, [5], and energy estimates for hyperbolic-parabolic systems, $[6,7]$ and references therein.

Recently, Nishida [9] announced a similar $L_{2}$ decay result for two equations of viscous barotropic gas. For two viscous conservation laws, there exists a coordinate of Riemann invariants which allows explicit computations. Kawashima has also announced the $L_{2}$ decay result for general system of hyperbolicparabolic type using his earlier works [2] and that of [5]. However, he does not give $L_{P}, p \neq 2, \infty$, description of the convergence. The pointwise description (1.26) is the natural one. Our analysis is carried out for (1.7), and therefore does not require the existence of a strictly convex entropy function. By studying the linear hyperbolic waves carefully, the rate of convergence for the general system (1.1) is the same as that for (1.7).

The rates obtained in the above theorems would be optimal if $\sigma=0$. If we view (1.20) as a perturbation of the linear system depending on diffusion waves $\theta$ :

instead of

$$
\frac{\partial v}{\partial t}+f^{\prime}(0) \frac{\partial v}{\partial x}+L(\theta) \frac{\partial v}{\partial x}=\frac{\partial^{2} v}{\partial x^{2}}
$$

$$
\frac{\partial v}{\partial t}+f^{\prime}(0) \frac{\partial v}{\partial x}=\frac{\partial^{2} v}{\partial x^{2}}
$$

as is done in the present paper, we would obtain optimal rates. This is, however, left to the future.

It is interesting to compare the similarity and differences in the large-time behavior between solutions of viscous conservation laws (1.1) and of hyperbolic conservation laws (1.8). Each mode for (1.1) depends only on one time-invariant $(1.23)_{4}$. For (1.8) each nonlinear mode has two time-invariants, while each linear mode has infinitely many time-invariants, see [4] and references therein. Moreover, the $L_{1}$-convergence rate to diffusion waves for solutions of (1.1) is around $t^{-1 / 2}$. On the other hand, the convergence rate to $N$-waves of solutions of 
(1.8) has been shown only to be $O(1) t^{-1 / 4}$ [4], for general systems. Although for the scalar hyperbolic conservation law the rate is still $t^{-1 / 2}$, for the general system $t^{-1 / 4}$ may be optimal. In other words, the nonlinear coupling of different modes for (1.8) may be substantially stronger than that for (1.1).

\section{Diffusion Waves}

We want to study the properties of diffusion waves. The first proposition relates the solution $\theta_{i}(x, t)$ of (1.11), (1.12) and $\theta_{i}^{*}$ of (1.23).

Proposition 2.1. Suppose that

$$
\delta \equiv \int_{-\infty}^{\infty}\left(\left|\theta_{i}(x, 0)\right|+\left|x \theta_{i}(x, 0)\right|\right) d x
$$

is bounded. Then $\theta(x, t)$ converges to $\theta^{*}$ time asymptotically in the sense that

$$
\left\|D^{l}\left[\theta_{i}(x, t)-t^{-1 / 2} \theta_{i}^{*}\left(\frac{x}{\sqrt{t}}\right)\right]\right\|_{L_{\mathbf{P}}}=O(1) \delta t^{\frac{1}{2 p}-\frac{l}{2}-1}
$$

for some $O(1)$ independent of $t$ and $\delta$. Moreover in the self-similar coordinate we have

$$
\begin{gathered}
\lim _{t \rightarrow \infty} \sqrt{t}\left\{\frac{l+1}{t^{2}}\left(D^{l} \theta_{i}\right)\left(\lambda_{i} t+\sqrt{t} \bar{x}, t\right)-D_{\bar{x}}^{l} \theta_{i}^{*}(\bar{x})\right\} \\
=\frac{1}{\sqrt{2 \pi}}\left(D^{l+1} e^{\left.-\frac{\bar{x}^{2}}{2}\right)} \int_{-\infty}^{\infty} y \theta_{i}(y, 0) d y, \quad \text { for } \quad b_{i}=0,\right. \\
\lim _{t \rightarrow \infty} \sqrt{t}\left\{\frac{l+1}{t^{2}}\left(D^{l} \theta_{i}\right)\left(\lambda_{i} t+\sqrt{t} \bar{x}, t\right)-D_{\bar{x}}^{l} \theta_{i}^{*}(\bar{x})\right\} \\
=-\frac{1}{b_{i}} \frac{2 \sqrt{2 \pi} \Delta_{i}}{\varphi_{i}^{0}(+\infty)-\varphi_{i}^{0}(-\infty)} D^{l+2}\left(\ln \psi_{i}(\bar{x})\right) \text { for } b_{i} \neq 0, \\
\Delta_{i} \equiv \int_{-\infty}^{0}\left(\varphi_{i}^{0}(y)-\varphi_{i}^{0}(-\infty)\right) d y+\int_{0}^{\infty}\left(\varphi_{i}^{0}(y)-\varphi_{i}^{0}(+\infty)\right) d y, \\
\varphi_{i}^{0}(y) \equiv \exp \left(-b_{i} \int_{i}^{y} \theta_{i}(\eta, 0) d y\right)
\end{gathered}
$$

uniformly in $\bar{x}$.

Proof. When $b_{i}=0$ we have from (1.13) and (1.23) that

$$
\begin{aligned}
e_{l}(x, t) & \equiv D^{l}\left[\theta_{i}(x, t)-t^{-1 / 2} \theta_{i}^{*}\left(\frac{x}{\sqrt{t}}\right)\right] \\
& =\frac{1}{\sqrt{2 \pi t}} D^{l} \int_{-\infty}^{\infty}\left[e^{-\frac{(x-y)^{2}}{2 t}}-e^{\left.-\frac{x^{2}}{2 t}\right] \theta_{i}(y, 0) d y}\right. \\
& =\frac{1}{\sqrt{2 \pi t}} \int_{-\infty}^{\infty} D^{l}\left[\int_{0}^{1} \frac{(x-s y)}{t} e^{\left.-\frac{(x-s y)^{2}}{2 t} d s\right] y \theta_{i}(y, 0) d y}\right. \\
& =\frac{1}{\sqrt{2 \pi t}} t^{-1-l / 2} \int_{-\infty}^{\infty}\left[\int_{0}^{1} p_{l+1}\left[\frac{(x-s y)}{t}\right] e^{-\frac{(x-s y)^{2}}{2 t}} d s\right] y \theta_{i}(y, 0) d y
\end{aligned}
$$


for some polynomial $p_{l+1}$ of degree $l+1$. Here we assume, for notational convenience that $\lambda_{i}=0$. From above, there exists $\gamma \in(0,1 / 2)$ such that

$$
\begin{aligned}
\left|e_{l}(x, t)\right| & \leqq \frac{1}{\sqrt{2 \pi}} t^{-1-l / 2} \int_{-\infty}^{\infty}\left[\int_{0}^{1} e^{-\gamma \frac{(x-s y)^{2}}{t}} d s\right]\left|y \theta_{i}(y, 0)\right| d y \\
& =\frac{1}{\sqrt{2 \pi}} t^{-1-l / 2} \int_{-\infty}^{\infty} e^{-\gamma \frac{(x-y)^{2}}{t}} \phi_{i}(y) d y, \\
\phi_{i}(y) & \equiv \int_{0}^{y}\left|\frac{y}{s^{2}} \theta_{i}\left(\frac{y}{s}, 0\right)\right| d s=\int_{ \pm \infty}^{y}\left|\theta_{i}(r, 0)\right| d r, \quad y \leqq 0 .
\end{aligned}
$$

Thus we have

$$
\begin{gathered}
\left\|\phi_{i}\right\|_{L_{1}}=\int_{-\infty}^{\infty}\left|y \theta_{i}(y, 0)\right| d y \leqq \delta \\
\left\|e_{l}(x, t)\right\|_{L_{p}}=\frac{1}{\sqrt{2 \pi}} t^{-1-l / 2}\left\|e^{-\frac{\gamma x^{2}}{t}}\right\|_{L_{p}}\left\|\phi_{i}\right\|_{L_{1}}=O(1) \delta t^{-1-l / 2+\frac{1}{2 p}} .
\end{gathered}
$$

This proves (i) for $b_{i}=0$.

For $b_{i} \neq 0$, and again, for simplicity, $\lambda_{i}=0$, we have from (1.14) and (1.23) that

$$
\begin{gathered}
\theta_{i}=\left(-\frac{1}{b_{i}} \ln \varphi\right)_{x}, \quad \varphi=\psi+\frac{1}{\sqrt{t}} E, \\
E=E_{1}+E_{2} \equiv \int_{0}^{\infty} e^{\frac{-(x-y)^{2}}{2 t}}\left(\varphi_{0}(y)-\varphi_{0}(\infty)\right) d y+\int_{-\infty}^{0} e^{\frac{-(x-y)^{2}}{2 t}}\left(\varphi_{0}(y)-\varphi_{0}(-\infty)\right) d y, \\
\varphi_{0}(y) \equiv \exp \left(-b_{i} \int \theta_{i}(\xi, 0) d \xi\right) .
\end{gathered}
$$

We note that there exists $\varepsilon_{0}>0$ such that

$$
\begin{gathered}
\varphi(x, t)=\frac{1}{\sqrt{2 \pi t}} \int_{-\infty}^{\infty} e^{\frac{-(x-y)^{2}}{2 t}} \varphi_{0}(y) d y \geqq \varepsilon_{0}, \\
\psi\left(\frac{x}{\sqrt{t}}\right)=\varphi_{0}(\infty) \int_{-\infty}^{\frac{x}{\sqrt{t}}} e^{-\frac{y^{2}}{2}} d y+\varphi_{0}(-\infty) \int_{\frac{x}{\sqrt{t}}}^{\infty} e^{-\frac{y^{2}}{2}} d y \geqq \varepsilon_{0} .
\end{gathered}
$$

From above we have

$$
\begin{gathered}
e_{l}(x, t)=D^{l}\left[\theta_{i}(x, t)-t^{-1 / 2} \theta_{i}^{*}\left(\frac{x}{\sqrt{t}}\right)\right]=D^{l+1}\left[\frac{1}{b_{i}} \ln \left(1+\frac{\zeta}{\sqrt{t}}\right)\right], \\
\zeta \equiv \frac{E}{\psi} .
\end{gathered}
$$

As noted above we have

$$
C_{0} \leqq 1+\frac{\zeta}{\sqrt{t}}=\frac{\varphi}{\psi} \leqq C_{1}
$$

for some positive constants $C_{1}$ and $C_{2}$. 
Thus we may apply the chain rule to yield

$$
\begin{aligned}
\left|D^{l+1} \ln \left(1+\frac{\xi}{\sqrt{t}}\right)\right| & =\sum_{\substack{1 \leqq \varrho \leqq l+1 \\
\alpha_{1}+\ldots+\alpha_{l+1}=\varrho}}\left|\frac{d^{\varrho} F}{d y^{\varrho}} C_{\varrho \alpha}\left(\frac{D \zeta}{\sqrt{t}}\right)^{\alpha_{1}} \ldots\left(\frac{D^{l+1} \zeta}{\sqrt{t}}\right)^{\alpha_{l+1}}\right| \\
& \leqq \sum_{\substack{\varrho, \alpha \\
\varrho \alpha}} C_{\varrho-1}(\varrho-1) !\left|1+\frac{\zeta}{\sqrt{t}}\right|^{-\varrho_{t}-\varrho / 2}|D \zeta|^{\alpha_{1}} \ldots\left|D^{l+1}\right|^{\alpha_{l+1}} \\
& =O(1) \sum t^{-\varrho / 2}|D \zeta|^{\alpha_{1}} \ldots\left|D^{l+1} \zeta\right|^{\alpha_{l+1}},
\end{aligned}
$$

where $F(y) \equiv \ln (1+y)$ and $C_{\varrho \alpha}$ are some constants. Consequently by Holder's inequality, we have

$$
\begin{aligned}
\left\|e_{l}\right\|_{L_{p}} & =O(1) \sum_{\varrho, \alpha} t^{-\varrho / 2}\left(\int_{-\infty}^{\infty}|D \zeta|^{\alpha_{i} p} \ldots\left|D^{l+1} \zeta\right|^{\alpha_{l+1} p} d x\right)^{1 / p} \\
& =O(1) \sum_{\varrho=1}^{l+1} t^{-\varrho / 2}\left(\sum_{\alpha} \prod_{j} \| D^{j \zeta} \frac{\|_{L_{p(l+1)}}^{\alpha_{j}}}{j}\right) .
\end{aligned}
$$

From the explicit form of $\psi$ we easily have

$$
\left|D^{j \zeta}\right|=O(1) \sum_{0 \leqq K \leqq j}\left|D^{k} E\right|\left|D^{j-k}\left(\frac{1}{\psi}\right)\right|=O(1) \sum_{0 \leqq K \leqq j}\left|D^{k} E\right| t^{\frac{-j+k}{2}} .
$$

Similar to the estimate for the case $b_{i}=0$, we have

$$
\begin{aligned}
\left|D^{k} E_{1}(x, t)\right| & =\mid \int_{0}^{\infty} t^{-k / 2} P_{k}\left(\frac{x-y}{\sqrt{t}}\right) e^{-\frac{(x-y)^{2}}{2 t}}\left(\phi_{0}(y)-\phi_{0}(\infty) d y \mid\right. \\
& \leqq t^{-k / 2} \int_{0}^{\infty} e^{-\frac{\gamma(x-y)^{2}}{t}} \mid\left(\phi_{0}(y)-\phi_{0}(\infty) \mid d y,\right.
\end{aligned}
$$

and so

$$
\left\|D^{k} E_{1}\right\|_{L_{q}}=O(1) t^{-\frac{k}{2}+\frac{1}{2 q}} \int_{-\infty}^{\infty}\left|\varphi_{0}(y)-\varphi_{0}(\infty)\right| d y .
$$

A similar estimate also holds for $E_{2}$. We conclude that

$$
\begin{gathered}
\left\|D^{j \zeta \|_{\frac{L_{p(l+1)}}{J}}}=O(1) \sum_{0 \leqq K \leqq j}\right\| D^{k} E \|_{\frac{L_{p(l+1)}}{J}} t^{\frac{-j+k}{2}}=O(1) \bar{\Delta} t^{-\frac{j}{2}+\frac{j}{2 p(l+1)}}, \\
\bar{\Delta} \equiv \int_{0}^{\infty}\left|\varphi_{0}(y)-\varphi_{0}(\infty)\right| d y+\int_{-\infty}^{0}\left|\varphi_{0}(y)-\varphi_{0}(-\infty)\right| d y \\
\left\|e_{l}\right\|_{L_{p}}=O(1)\left(\bar{\Delta}+t^{-1 / 2} \bar{\Delta}^{2}+\ldots+t^{-l / 2} \bar{\Delta}^{l+1}\right) t^{-\frac{l}{2}-1+\frac{1}{2 p}}
\end{gathered}
$$

where we have used

$$
\sum j \alpha_{j}=l+1 \text {. }
$$

Since $\bar{\Delta}=O(1) \delta$, this proves (i) for $b_{i} \neq 0$. (ii) and (iii) are proved using a similar method and the dominated convergence theorem. Q.E.D. 
Proposition 2.2. Under the same assumption as Proposition 2.1 we have

(ii) $\left\|D^{l}\left(\theta_{i}(t) \theta_{j}(t)\right)\right\|_{L_{1}}=O(1) \delta^{2}(1+t)^{-l / 2-1}, \quad i \neq j, \quad i, j=1,2, \ldots, n$,

(iii) $\left\|D^{l}\left(\theta_{i}(t) \theta_{j}(t)\right)\right\|_{L_{2}}=O(1) \delta^{2}(1+t)^{-l / 2-5 / 4}, \quad i \neq j, \quad i, j=1,2, \ldots, n$.

Proof. (i) follows easily from (i) of Proposition 2.1 and that it holds clearly for $\theta=\theta_{*}$. Similarly, to prove (ii) and (iii) we need only to show that they hold for $\theta_{i}^{*}(\bar{x}) \theta_{j}^{*}(\bar{x}) t^{-1}$. In fact by explicit computations, it is easy to see that $\theta_{i}^{*}(\bar{x}) \theta_{j}^{*}(\bar{x}) t^{-1}$ decays exponentially in $t$. This proves the proposition. Q.E.D.

\section{3. $L_{2}$ Decay}

To prove Theorem 1.1 on $L_{2}$ decay we view (1.20) as a perturbation of a linear system with constant coefficient

$$
\frac{\partial v}{\partial t}+f^{\prime}(0) \frac{\partial v}{\partial t}=\frac{1}{2} \frac{\partial^{2} v}{\partial x^{2}}-F, \quad F \equiv L(\theta) v_{x}+N\left(\theta, v_{x}\right)
$$

With zero data (1.21) we have

$$
\begin{gathered}
v(x, t)=-\int_{0}^{t} \int_{-\infty}^{\infty} G(x-y, t-s) F(y, s) d y d s \\
G(x, t) \equiv \frac{1}{\sqrt{2 \pi t}}\left(\begin{array}{ccc}
e^{-\frac{\left(x-\lambda_{1} t\right)^{2}}{2 t}} & \ddots & 0 \\
0 & e^{-\frac{\left(x-\lambda_{n} t\right)^{2}}{2 t}}
\end{array}\right) .
\end{gathered}
$$

Lemma 3.1. There exist constant $\eta>0, c>0$ such that for $|\theta|_{\infty}+\left|v_{y}\right|_{\infty} \leqq \eta$,

$$
\begin{aligned}
& \left\|D^{l-1}\left(L(\theta) v_{y}\right)\right\|_{L_{1}} \leqq c\left(\|\theta\|_{L_{2}}\left\|D^{l} v\right\|_{L_{2}}+\left\|D^{l-1} \theta\right\|_{L_{2}}\left\|v_{y}\right\|_{L_{2}}\right), \\
& \left\|D^{l}\left(L(\theta) v_{y}\right)\right\|_{L_{2}} \leqq c\left(|\theta|_{\infty}\left\|D^{l+1} v\right\|_{L_{2}}+\left\|D^{l} \theta\right\|_{L_{2}}\left|v_{y}\right|_{\infty}\right), \\
& \left\|D^{l-1} N\left(\theta, v_{y}\right)\right\|_{L_{1}} \leqq c\left(\sum_{i \neq j}\left\|D^{l-1}\left(\theta_{i} \theta_{j}\right)\right\|_{L_{1}}+\left\|D^{l} v\right\|_{L_{2}}\right. \\
& \times\|D v\|_{L_{2}}+\left(\|\theta\|_{L_{2}}+\left\|v_{y}\right\|_{L_{2}}\right) \\
& \left.\times\left(|\theta|_{\infty}+\left|v_{y}\right|_{\infty}\right)\left(\left\|D^{l-1} \theta\right\|_{L_{2}}+\left\|D^{l} v\right\|_{L_{2}}\right)\right), \\
& \left\|D^{l} N\left(\theta, v_{y}\right)\right\|_{L_{2}} \leqq c\left(\sum_{i \neq j}\left\|D^{l}\left(\theta_{i} \theta_{j}\right)\right\|_{L_{2}}+\left|v_{y}\right|_{\infty}\left\|D^{l+1} v\right\|_{L_{2}}\right. \\
& \left.+\left(|\theta|_{\infty}+\left|v_{y}\right|_{\infty}\right)^{2}\left(\left\|D^{l} \theta\right\|_{L_{2}}+\left\|D^{l+1} v\right\|_{L_{2}}\right)\right) .
\end{aligned}
$$

Proof. The lemma can be proved easily via Moser's inequalities which are listed in Lemmas A2-A4 of the Appendix. We will prove (iii), (iv) for demonstration. The nonlinear function $N$ can be written as

$$
\begin{gathered}
N(a, b)=\frac{1}{2} \sum_{i \neq j}^{n}\left(f^{\prime \prime}(0) r_{i}, r_{j}\right) a_{i} a_{j}+\frac{1}{2}\left(f^{\prime \prime}(0) b, b\right)+H(a, b), \\
H(a, b)=O(1)(|a|+|b|)^{3} .
\end{gathered}
$$


By Lemmas A2-A4,

$$
\begin{aligned}
& \left\|D^{l-1} N\left(\theta \cdot v_{y}\right)\right\|_{L_{1}} \leqq c\left(\sum_{i \neq j}\left\|D^{l-1}\left(\theta_{i} \theta_{j}\right)\right\|_{L_{1}}+\left\|D^{l-1}\left(v_{y}^{2}\right)\right\|_{\mathrm{L}_{1}}\right. \\
& \left.+\left\|D^{l-1} H\left(\theta, v_{y}\right)\right\|_{L_{1}}\right), \\
& \left\|D^{l-1}\left(v_{y}^{2}\right)\right\|_{L_{1}} \leqq c\left(\|D v\|_{L_{2}}\left\|D^{l} v\right\|_{L_{2}}\right), \\
& \left\|D^{l-1} H\left(\theta, v_{y}{ }^{2}\right)\right\|_{L_{1}} \leqq c\left(|\theta|_{\infty}+\left|v_{y}\right|_{\infty}\right)\left(\|\theta\|_{L_{1}}+\left\|v_{y}\right\|_{L_{2}}\right) \\
& \left.\times\left(\left\|D^{l-1} \theta\right\|_{L_{2}}+\| D^{l} v\right) \|_{L_{2}}\right), \\
& \left\|D^{l} H\left(\theta, v_{y}\right)\right\|_{L_{2}} \leqq c\left(\sum_{i \neq j}\left\|D^{l}\left(\theta_{i} \theta_{j}\right)\right\|_{L_{2}}+\left\|D^{l}\left(v_{y}\right)^{2}\right\|_{L_{2}}\right. \\
& \left.+\left\|D^{l} H\left(\theta, v_{y}\right)\right\|_{L_{2}}\right), \\
& \left\|D^{l}\left(v_{y}\right)^{2}\right\|_{L_{2}} \leqq c\left|v_{y}\right|_{\infty}\left\|D^{l+1} v\right\|_{L_{2}}, \\
& \left\|D^{l} H\left(\theta, v_{y}\right)\right\|_{L_{2}} \leqq c\left(|\theta|_{\infty}+\left|v_{y}\right|_{\infty}\right)^{2}\left(\left\|D^{l} \theta\right\|_{L_{2}}+\left\|D^{l+1} v\right\|_{L_{2}}\right) .
\end{aligned}
$$

This proves (iii), (iv). Q.E.D.

The following lemma follows from easy computations.

Lemma 3.2. Let $\alpha, \beta, \gamma$ be positive constants. Then

$$
\int_{0}^{t / 2}(1+t-s)^{-\beta}(1+s)^{-\gamma} d s=O(1)(1+t)^{-\alpha}
$$

if $\alpha \leqq \beta, \alpha \leqq \beta+\gamma-1, \gamma \neq 1$, or if $\alpha<\beta, \alpha \leqq \beta+\gamma-1, \gamma=1$,

$$
\int_{t / 2}^{t}(1+t-s)^{-\beta}(1+s)^{-\gamma} d s=O(1)(1+t)^{-\alpha},
$$

if $\alpha \leqq \gamma, \alpha \leqq \beta+\gamma-1, \beta \neq 1$, or if $\alpha<\gamma, \alpha \leqq \beta+\gamma-1, \beta=1$,

$$
\int_{0}^{t} e^{-\beta(t-s)}(1+s)^{-\gamma} d s=O(1)(1+t)^{-\alpha},
$$

if $\alpha \leqq \gamma$.

Lemma 3.3 (Energy estimate). Suppose that (1.20), (1.21) has a solution $v(x, t)$ in $C\left([0, T], H^{m+1}\right) \cap L_{2}\left([0, T], H^{m+2}\right)$. Then there exists $c>0$ independent of $t \in[0, T]$ such that

$$
\begin{aligned}
& \frac{\partial}{\partial t}\left(\left\|D^{l} v\right\|_{L_{2}}^{2}+\frac{1}{4}\left\|D^{l+1} v\right\|_{L_{2}}^{2}\right) \\
& \quad \leqq c\left(\left(|\theta|_{\infty}+\left|v_{x}\right|_{\infty}\right)^{2}\left\|D^{l} v\right\|_{L_{2}}^{2}+\sum_{i \neq j}\left\|D^{l}\left(\theta_{i} \theta_{j}\right)\right\|_{L_{2}}^{2}\right. \\
& \left.\quad+\left(|\theta|_{\infty}+\left|v_{x}\right|_{\infty}\right)^{2}\left\|D^{l} \theta\right\|_{L_{2}}^{2}\right), \quad 0 \leqq t \leqq T, \quad 0 \leqq l \leqq m+1,
\end{aligned}
$$

provided that $|\theta|_{\infty}+\left|v_{x}\right|_{\infty} \leqq \eta$, where $\eta$ is defined in Lemma 3.1.

The above lemma follows from the usual energy method of integrating $\left(D^{l} v\right) \cdot D^{l}((1.20))$ over $x$ and using (ii), (iv) of Lemma 3.1. The next lemma is proved by the spectral method, [2]. 
Lemma 3.4. Set

$$
\tilde{v}(x, t) \equiv \int_{-\infty}^{\infty} G(x-y, t) \tilde{w}(y) d y .
$$

Then there exist $c>0, \beta>0$ such that

$$
\begin{gathered}
\left\|D^{l} \tilde{v}(t)\right\|_{L_{2}} \leqq\left(e^{-\beta t}\left\|D^{l} \tilde{w}\right\|_{L_{2}}\right)+c(1+t)^{-\frac{1}{4}-\frac{l}{2}+\frac{k}{2}}\left\|D^{k} \tilde{w}\right\|_{L_{1}}, \\
0 \leqq k \leqq l, \quad t \geqq 0 .
\end{gathered}
$$

With these lemmas we are ready to prove the main decay estimates.

Proposition 3.5. Suppose that $\delta$ of Theorem 1.1 is sufficiently small and that the solution $v(x, t)$ of $(1.20),(1.21)$ exists in $c\left([0, \infty], H^{m+1}\right) \cap L^{2}\left([0, \infty), H^{m+2}\right)$. Then

$$
\left\|D^{l} v(t)\right\|_{L_{2}}=O(1) \delta(1+t)^{-\frac{1}{4}-\frac{l}{2}+\sigma}, \quad 0 \leqq l \leqq m+1,
$$

for all $\sigma>0$ sufficiently small.

Proof. Fix $T>0$ and set

$$
M_{l} \equiv M_{l}(T) \equiv \sup _{0 \leqq t \leqq T}(1+t)^{\frac{1}{4}+\frac{l}{2}-\sigma}\left\|D^{l} v(t)\right\|_{L_{2}}, \quad 0 \leqq l \leqq m+1 .
$$

From (3.2), Lemma 3.4,

$$
\begin{aligned}
\left\|D^{l} v(t)\right\|_{L_{2}} & =O(1)(\mathrm{I}+\mathrm{II}+\mathrm{III}), \\
\mathrm{I} & \equiv \int_{0}^{t} e^{-\beta(t-s)}\left\|D^{l}\left(L(\theta) v_{y}+N\left(\theta, v_{y}\right)\right)(s)\right\|_{L_{2}} d s, \\
\mathrm{II} & \equiv \int_{0}^{t / 2}(1+t-s)^{\frac{1}{4}-\frac{l}{2}}\left\|\left(L(\theta) v_{y}+N\left(\theta, v_{y}\right)\right)(s)\right\|_{L_{1}} d s, \\
\mathrm{III} & \equiv \int_{t / 2}^{t}(1+t-s)^{-\frac{1}{4}-\frac{1}{2}}\left\|D^{l-1}\left(L(\theta) v_{y}+N\left(\theta, v_{y}\right)\right)(s)\right\|_{L_{1}} d s .
\end{aligned}
$$

For I we apply (ii), (iv) of Lemma 3.2

$$
\begin{aligned}
\mathrm{I}= & O(1) \int_{0}^{t} e^{-\beta(t-s)}\left(|\theta|_{\infty}\left\|D^{l+1} v\right\|_{L_{2}}\right)+\left\|D^{l} \theta\right\|_{L_{2}}\left|v_{y}\right|_{\infty}+\sum_{i \neq j}\left\|D^{l}\left(\theta_{i} \theta_{j}\right)\right\|_{L_{2}} \\
& +\left|v_{y}\right|_{\infty}\left\|D^{l+1} v\right\|_{L_{2}}+\left(|\theta|_{\infty}+\left|v_{y}\right|_{\infty}\right)^{2}\left(\left\|D^{l} \theta\right\|_{L_{2}}+D^{l+1} v \|_{L_{2}}\right)(s) d s .
\end{aligned}
$$

Except the terms involving $D^{l+1} v$, the right-hand side above is estimated using Proposition 2.2 and (3.3). We use Lemma 3.3 to deal with $D^{l+1} v$ :

$$
\begin{aligned}
& \int_{0}^{t} e^{-\beta(t-s)}\left\|D^{l+1} v\right\|_{L_{2}}(s) d s=O(1) \int_{0}^{t} e^{-\beta(t-s)}\left\|D^{l} v(s)\right\|_{L_{2}}^{2} d s \\
& +O(1) \int_{0}^{t} e^{-\beta(t-s)}\left[\left(|\theta|_{\infty}+\left|v_{y}\right|_{\infty}\right)^{2}\left\|D^{l} v\right\|_{L_{2}}^{2}\right. \\
& \left.+\sum_{i \neq j}\left\|D^{l}\left(\theta_{i} \theta_{j}\right)\right\|_{L_{2}}^{2}+\left(|\theta|_{\infty}+\left|v_{y}\right|_{\infty}\right)^{2}\left\|D^{l} \theta\right\|_{L_{2}}^{2}\right](s) d s,
\end{aligned}
$$


where we have also used (1.21). We use (3.2) and the Sobolev inequality to yield

$$
\begin{aligned}
|\theta(s)|_{\infty}+\left|v_{y}(s)\right|_{\infty} & =O(1)(1+s)^{-1 / 2}\left(\delta+M_{1}+M_{2}\right), \\
\left\|D^{l} v(s)\right\|_{L_{2}} & =O(1)(1+s)^{-\frac{1}{4}-\frac{l}{2}+\sigma} M_{l},
\end{aligned}
$$

and so from (iii) of Lemma 3.2 and Proposition 2.2,

$$
\begin{aligned}
& \left(\int_{0}^{t} e^{-\beta(t-s)}\left\|D^{l+1} v\right\|_{L_{2}}^{2}(s) d s\right)^{1 / 2} \\
& \quad=O(1)(1+t)^{-\frac{1}{4}-\frac{l}{2}+\sigma}\left\{\delta^{2}+M_{1}+M_{2}+M_{l}\right\}, \quad 0 \leqq t \leqq T .
\end{aligned}
$$

Similarly from Proposition 2.2, Lemmas 3.1 and 3.2 that

$$
\begin{aligned}
& \mathrm{I}=O(1)(1+t)^{-\frac{1}{4}-\frac{l}{2}+\sigma}\left(\delta+M_{1}+M_{2}\right)\left(\delta+M_{l}\right), \\
& \mathrm{II}=O(1)(1+t)^{-\frac{1}{4}-\frac{l}{2}+\sigma}\left(\delta M_{1}+\delta^{2}+M_{1}^{2}+\left(\delta+M_{1}\right)^{2}\left(\delta+M_{1}+M_{2}\right)\right), \\
& \mathrm{III}=O(1)(1+t)^{-\frac{1}{4}-\frac{l}{2}+\sigma}\left(\left(\delta+M_{1}+M_{2}\right)\left(\delta+M_{l}\right)\right) .
\end{aligned}
$$

We conclude from (3.4) that

$$
M=O(1)\left(\delta+(\delta+M)^{2}+(\delta+M)^{3}\right), \quad M \equiv \sum_{l=0}^{m+1} M_{l}(T) .
$$

It follows from (3.5) that for $\delta$ sufficiently small $M=O(1) \delta$. This proves the proposition. Q.E.D.

Finally, the proof of Theorem 1.1 follows from Proposition 3.5 and the standard local existence theorem for uniform parabolic systems.

\section{Asymptotic Behavior}

We first prove Theorem 1.2 for $p=\infty$. From (1.18) we need to show that

$$
\left|D^{l+1} v(t)\right|_{\infty}=O(1) \delta t^{-\frac{l}{2}-1+\sigma}, \quad 0 \leqq l \leqq m-1 .
$$

This follows immediately from Theorem 1.1 and the Sobolev inequality

$$
\begin{aligned}
\left|D^{l+1} v(t)\right|_{\infty} & =O(1)\left\|D^{l+1}(t)\right\|_{L_{2}}^{1 / 2}\left\|D^{l+2}(t)\right\|_{L_{2}}^{1 / 2} \\
& =O(1) \delta(1+t){ }^{\left(-\frac{1}{4}-\frac{l+1}{2}+\sigma-\frac{1}{4}-\frac{l+2}{2}+\sigma\right) \frac{1}{2}}=O(1) t^{-\frac{l}{2}-1+\sigma} .
\end{aligned}
$$

The estimate (1.26) follows from this estimate and Proposition 2.1. Next we prove Theorem 1.2 for $p=1$. From Proposition 2.1, (1.18) and (3.2) we have

$$
\begin{aligned}
\left\|D^{l}\left(u-\theta^{*}\right)\right\|_{L_{p}}(t)= & \left\|D^{l+1} v\right\|_{L_{p}}(t) \\
\leqq & \left(\int_{-\infty}^{\infty}\left|\int_{0}^{t / 2} \int_{-\infty}^{\infty}\left(D^{l+1} G\right)(x-y, t-s) F(y, s) d y d s\right|^{p} d x\right)^{1 / p} \\
& +\left(\int_{-\infty}^{\infty}\left|\int_{t / 2}^{t}\left(D^{l+1} G\right)(y, s) F(x-y, t-s) d y d s\right|^{p} d x\right)^{1 / p} .
\end{aligned}
$$


From Proposition 2.2, Lemma 3.1 and Theorem 1.1 and the above estimate on $L_{\infty}$ decay, we have

$$
\left\|D^{i} F(s)\right\|_{L_{1}}=O(1) \delta(1+s)^{-1-\frac{i}{2}+\sigma}, \quad i=0,1, \ldots, m, \quad s \geqq 0 .
$$

By the explicit form of $G$, a similar decay also holds for $G$ :

$$
\left\|D^{i+1} G(s)\right\|_{L_{1}}=O(1)(1+s)^{-\frac{1}{2}-\frac{i}{2}}, \quad i=0,1, \ldots, m, \quad s \geqq 0 .
$$

Thus we have from the usual estimates for convolution that

$$
\begin{aligned}
\left\|D\left(u-\theta^{*}\right)\right\|_{L_{1}}(t) \leqq & \int_{0}^{t / 2}\left\|D^{l+1} G(t-s)\right\|_{L_{1}}\|F(s)\|_{L_{1}} d s \\
& +\int_{t / 2}^{t}\|D G(s)\|_{L_{1}}\left\|D^{l} F(t-s)\right\|_{L_{1}} d s \\
= & O(1) \delta \int_{0}^{t / 2}(1+t-s)^{-\frac{1}{2}-\frac{l}{2}}(1+s)^{-1+\sigma} d s \\
= & O(1) \delta \int_{t / 2}^{t}(1+s)^{-1-\frac{l}{2}+\sigma}(1+t-s)^{-1 / 2} d s .
\end{aligned}
$$

This implies Theorem 1.2 for $p=1$ by virtue of Lemma 3.2.

It remains to prove Theorem 1.2 for $p \neq 1, p \neq 2, p \neq \infty$. This follows from the usual interpolation formulas:

$$
\begin{array}{ll}
\|f\|_{L_{p}} \leqq\|f\|_{L_{2}}^{2 / p}|f|_{\infty}^{\frac{p-2}{p}}, & 2 \leqq p \leqq \infty, \\
\|f\|_{L_{p}} \leqq\|f\|_{L_{1}}^{2 / p}\|f\|_{L_{2}}^{2-\frac{2}{p}}, & 1 \leqq p \leqq 2 .
\end{array}
$$

This completes the proof of Theorem 1.2.

\section{General System}

In this section we indicate how to generalize our analysis to systems of the general form (1.1) when the viscosity $B(u)$ is not the identity matrix and (1.1) is not uniformly parabolic but hyperbolic-parabolic. Most physical models such as the compressible Navier-Stokes equations have such properties. First, we follow the approach of Sects. 3, 4, and 6 of [6] in deriving the asymptotic state. The asymptotic state consists of diffusion waves $\psi$ (with slightly a different construction from $\theta$ in Sect. 2 of the present article) and a linear hyperbolic wave $\xi$. Write

$$
u=\psi+\xi+u^{*}, \quad v(x, t) \equiv \int_{-\infty}^{x} u^{*}(y, t) d y, \quad v( \pm \infty, t)=0, \quad t \geqq 0,
$$

so that $v$ satisfies cf. (6.29) of [6],

$$
\begin{aligned}
v_{t}+f^{\prime}(\psi) v_{x}= & B\left(v_{x}+\psi+\xi\right)\left(v_{x x}+\xi_{x}\right)+O(1) \\
& \times\left(\left|v_{x}\right|^{2}+|\xi|+\left|\psi_{x}\right|^{2}+\left(\left|v_{x}\right|+\xi\right)\left|\psi_{x}\right|+\sum_{i \pm j}\left|\psi_{i x} \psi_{j x}\right|\right)
\end{aligned}
$$


The system is then viewed as a perturbation of a linear system

$$
v_{t}+f^{\prime}(0) v_{x}=B(0) v_{x x}+\tilde{F},
$$

cf. $(2.20)^{\prime}$. The next step is to use the Fourier transform to study the decay property of the solution operator of the linear system

$$
w_{t}+f^{\prime}(0) w_{x}=B(0) w_{x x} .
$$

We then apply the argument in the proof of Theorem 4.1. If the case (1.1) is uniformly parabolic there exists an energy estimate similar to (4.7). The situation is somewhat more complicated here because $\widetilde{F}$ depends on $v, D v$, and $D^{2} v$; while $F$ in (2.20)' depends only on $v$ and $D v$. Nevertheless, we need only to apply the energy estimate twice to express $D^{l+2} v$ in terms of $D^{l} v$. When (1.1) is not uniformly parabolic one needs to use the particular coupling property of the equations in the system to derive the energy estimates, cf. [6] and references therein. Finally, $\widetilde{F}$ also depends on the linear hyperbolic wave $\xi$. This causes no problem because $\xi$ has higher rates of decay than the diffusion wave $\psi$, Sect. 7 of [6].

\section{Appendix}

Lemma $\mathbf{A} 1$ (Sobolev inequality). If $v \in H^{\prime}(\mathbb{R})$, then

$$
|v|_{\infty} \leqq c|v|_{L_{2}}^{1 / 2}\|D v\|_{L_{2}}^{1 / 2}
$$

for some constant $c$.

Lemmas A2 and A3 are Moser's inequalities [3].

Lemma A2. If $u, v \in H^{l} \cap L^{\infty}$, then

$$
\begin{aligned}
& \left\|D^{l}(u v)\right\|_{L_{2}} \leqq c\left(|u|_{\infty}\left\|D^{l} v\right\|_{L_{2}}+|v|_{\infty}\left\|D^{l} u\right\|_{L_{2}}\right), \\
& \left\|D^{l}(u v)\right\|_{L_{1}} \leqq c\left(\|u\|_{L_{2}}\left\|D^{l} v\right\|_{L_{2}}+\|v\|_{\infty}\left\|D^{l} u\right\|_{L_{2}}\right) .
\end{aligned}
$$

Lemma A3. Suppose $N$ is a smooth function in the neighborhood of $O \in \mathbb{R}^{N}$. Suppose $v$ is a $\mathbb{R}^{N}$-valued function $v \in H^{l} \cap L^{\infty}$. Then $\exists$ constant $\eta$ depending on $N$ and constant $c(\eta)$ such that

$$
\left\|D^{l} N(v)\right\|_{L_{2}} \leqq c(\eta)\left\|D^{l} v\right\|_{L_{2}}
$$

provided $|v|_{\infty} \leqq \eta$

Lemma A4. Suppose $H$ is a smooth function in the neighborhood of $O \in \mathbb{R}^{N}$, and $H(b)$ $=O\left(|b|^{p}\right)$ near $b=0$. Suppose $v$ is a $\mathbb{R}^{N}$-valued function, $v \in H^{l} \cap L^{\infty}$. Then $\exists$ constant $\eta$ and $c$, as in Lemma $A 3$ such that

$$
\begin{aligned}
& \left\|D^{l} H(v)\right\|_{L_{2}} \leqq c(n)|v|_{\infty}^{p-1}\left\|D^{l} v\right\|_{L_{2}}, \\
& \left\|D^{l} H(v)\right\|_{L_{1}} \leqq c(n)|v|_{\infty}^{p-2}\|v\|_{L_{2}}\left\|D^{l} v\right\|_{L_{2}} .
\end{aligned}
$$

Proof. This is a direct consequence of Lemmas A 2 and A3. 


\section{References}

1. Hopf, E.: The partial differential equation $u_{t}+u u_{x}=\mu u_{x x}$. Commun. Pure Appl. Math. 3, 201-230 (1950)

2. Kawashima, S.: Systems of a hyperbolic-parabolic composite type, with applications to the equations of magnetohydrodynamics. Doctoral Thesis, Kyoto University 1983

3. Lax, P.D.: Hyperbolic systems of conservation laws. II. Commun. Pure Appl. Math. 10, 537-566 (1957)

4. Liu, T.-P.: Pointwise convergence to $\mathrm{N}$-waves for solutions of hyperbolic conservation laws. Bull. Inst. Math. Academia Sinica, 1987

5. Liu, T.-P.: Nonlinear stability of shock waves for viscous conservation laws. Mem. Am. Math. Soc. 328, 1-108 (1985)

6. Liu, T.-P.: Shock waves for compressible Navier-Stokes equations are nonlinearly stable. Commun. Pure Appl. Math. 39, 565-594 (1986)

7. Matzumura, A., Nishida, T.: The initial value problem for the equations of motion of viscous and heat-conductive gases. J. Math. Kyoto Univ. 20, 67-104 (1980)

8. Moser, J.: A rapidly convergent iteration method and nonlinear partial differential equation. I. Ann. Sc. Norm. Super. Pisa 20, 265-315 (1966)

9. Nishida, T.: Asymptotic behavior of solutions of equation for viscous gas motion (preprint)

Communicated by A. Jaffe

Received October 27, 1986; in revised form December 29, 1986 
